\title{
The Prime Spectra of Regular Rings
}

\begin{tabular}{|c|c|c|}
\hline Nazar H. Shuker & Abdul Aali J. Mohammad & Huda Y. Khalil \\
\hline & & Huda196079@gmail.com \\
\hline $\begin{array}{l}\text { llege of Computer } \\
\text { iences and Mathematic }\end{array}$ & $\begin{array}{l}\text { College of Education } \\
\text { cs }\end{array}$ & $\begin{array}{c}\text { College of Basic } \\
\text { Education }\end{array}$ \\
\hline
\end{tabular}

Received on: 11/10/2006

Accepted on: 24/12/2006

ABSTRACT

In this work, we study the prime spectrum of regular rings. Also we study some topological concepts as quasi-compact, compact, totally disconnected, and irreducible topological space in order to prove some new results on the prime spectrum of regular ring.

Keywords: Prime ideals; Prime spectrum; Zariski topology; Open sets; Regular rings.

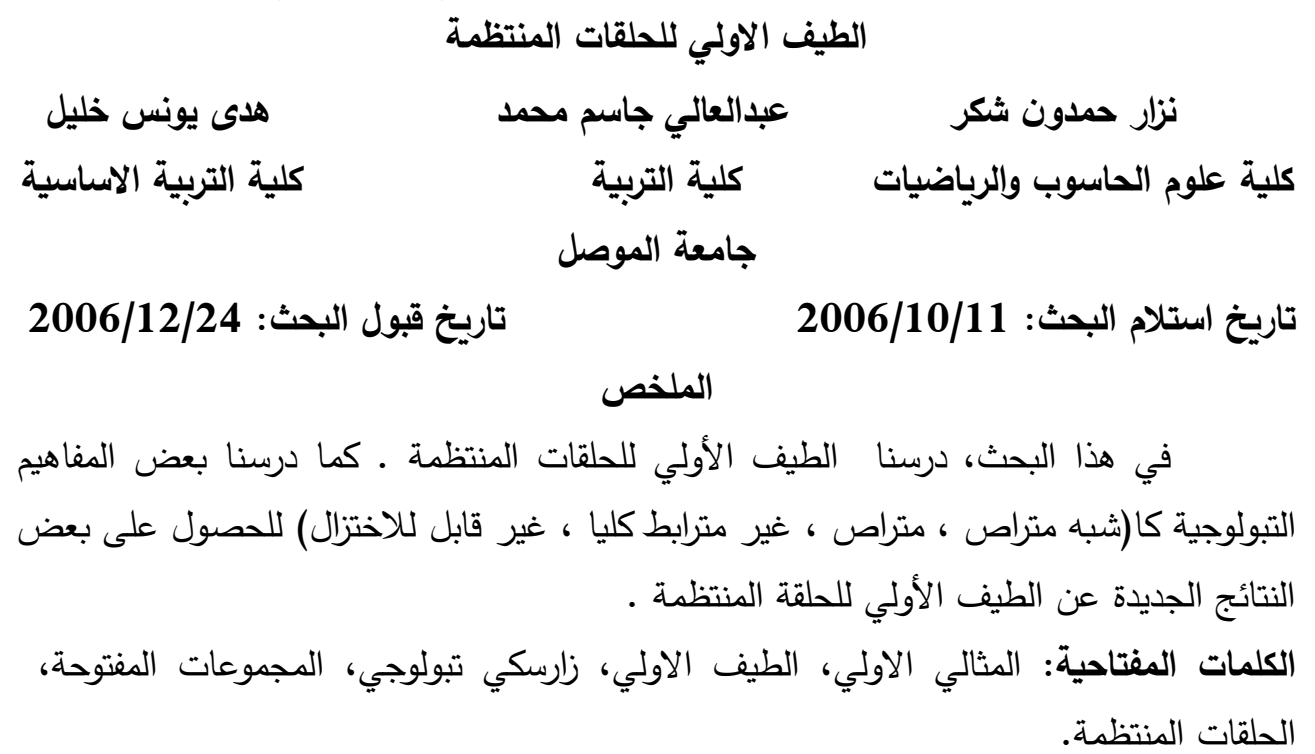

\section{Introduction:}

In this work, all rings are assumed to be commutative with identity. Let $R$ be a ring. The set of all prime ideals of $R$ is called the prime spectrum of $R$ and is denoted by $\operatorname{Spec}(R)$. The prime spectrum of rings has been studied by many mathematician like Heinzer and Roitman [6], and Lemmermeyer [10]. The topology on $\operatorname{Spec}(R)$ is called the Zariski topology. 
For every ideal $I$ of $R$ we denote by $V(I)$ the subset of $\operatorname{Spec}(R)$ consisting of all prime ideals that contain $I$.

For every ideal $I$ of $R$ we write $U(I)=\operatorname{Spec}(R) / V(I)$ for the set of prime ideals not containing $I$. The collection of subset $U(I)$ of $\operatorname{Spec}(R)$ for all ideals $I$ in $R$ form a topological space with open sets $U(I)$. The closed sets of the topology are the sets $V(I)$ for all ideals $I$ in $R$.

A ring $R$ is called a regular ring if for every $a$ in $R$ there exists $b$ in $R$ such that $a=a b a$. Regular rings are studied by many authors in recent years, see for example, Fisher [5], and Ming [11], [12], [13] .

\section{Open Sets in Spectrum of Regular Rings and Homeomorphism:}

In this section, we give some basic properties of open sets in the spectrum of regular ring. We prove that the spectrum topology of regular ring is homeomorphic to the spectrum topology of $S$.

\section{Proposition 2.1:[10]}

Suppose that $R$ is a ring, $I$ and $J$ are ideals of $R$, then:

i. $J \subseteq I$ implies that $V(I) \subseteq V(J)$

ii. $V(0)=\operatorname{Spec}(R), V(1)=\phi$

\section{Proposition 2.2:[1]}

Let $R$ be a regular ring. Then $R$.of Ifor every ideal $I=\sqrt{I}$

\section{Definition 2.3:[14]}

The open sets are $\{U(I): I$ is an ideal of $R\}$, where $U(I)$ is the complement of $V(I)$.

\section{Proposition 2.4:}

Let $R$ be a regular ring. Then

$U(I)=U(J)$ if and only if $I=J$

Proof:

If $I=J$, then obviously $U(I)=U(J)$

Conversely, assume that $U(I)=U(J)$

then $V(I)=V(J)$.By Proposition (2.1) we have

$V(I) \subseteq V(J) \Leftrightarrow \sqrt{J} \subseteq \sqrt{I}$,

$V(J) \subseteq V(I) \Leftrightarrow \sqrt{I} \subseteq \sqrt{J}$, since $V(I)=V(J)$ 
Therefore $\sqrt{I}=\sqrt{J}$.

Since $R$ is regular then by Proposition (2.2)

$I=\sqrt{I}$ and $J=\sqrt{J}$

Thus $I=J$.

Now, we prove the following theorem which gives a connection between open sets and radical ideal.

\section{Theorem 2.5:}

Let $R$ be a ring and $a, b \in R$. Then

$U(a)=U(b)$ if and only if $\sqrt{R a}=\sqrt{R b}$.

\section{Proof:}

Let $U(a)=U(b)$, since $V(a)$ is the complement of $U(a)$, then $V(a)=V(b)$.

Now, $\sqrt{R a}$ is an ideal generated by $a$, implies that

$\sqrt{R a}=\sqrt{(a)}$

$=\cap\{P:(a) \subseteq P\}, P$ is a prime ideal

$=\bigcap\{P: P \in V(a)\}$

$=\bigcap\{P: P \in V(b)\}$ since $V(a)=V(b)$

$=\cap\{P:(b) \subseteq P\}$

$=\sqrt{(b)}=\sqrt{R b}$

Conversely, let $\sqrt{R a}=\sqrt{R b}$. Then, $\sqrt{(a)}=\sqrt{(b)}$, this implies that $V((a))$ $=V((b))$, where $(a)$ is an ideal generated by $a$.

Now, if $P \in V(a)$ then $a \in P$. Thus $(a) \subset P$ since $(a)$ is the smallest ideal containing $a$. Therefore $P \in V((a))$. If $P \in V((a))$, then $a \in(a) \subset P$.

So $P \in V(a)$. Hence $V(a)=V((a))$. This implies that $V(a)=V(b)$.

But $U(a), U(b)$ is a complement of $V(a), V(b)$ therefore $U(a)=U(b)$.

\section{Proposition 2.6:}

Let $R$ be a regular ring, $a, b, \in R$, then $U(a)=U(b)$ if and only if $R a=R b$.

Proof:

Assume that . $V(a)=V(b)$ that is $U(a)=U(b)$

By Theorem (2.5) we get 
$\sqrt{R a}=\sqrt{R b}$.

By regularity we have $R a=\sqrt{R a}$.

Therefore $R a=R b$.

The converse is obvious.

Next, we give the following result .

\section{Theorem 2.7 :}

If $R$ is a regular ring, then for all $a \in R$, there exists an idempotent $e$ such that $U(a)=U(e)$.

Proof:

Let $a \in R$, then there exists $b \in R$ such that $a=a b a$, put $e=b a$ so we have $a=a e$.

Let $P \in U(a)$ then $a \notin P$ therefore $a e \notin P$. If $e \in P$, then $1-e \notin P$ so $a(1-e) \notin P$ which implies that $0 \notin P$, which is a contradiction. Hence $e \notin P$, therefore $P \in U(e)$ that is $U(a) \subseteq U(e)$.

Now, let $P \in U(e)$, then $e \notin P$ therefore $a b \notin P$. If $a \in P$,then $a b \in P$,contradiction.

So $a \notin P$;therefore $P \in U(a)$ and $U(e) \subseteq U(a)$.

Hence, $U(e)=U(a)$.

\section{Definition 2.8:}

Let $R$ be a ring and $S$ be a subset of $R$ such that $S=\left\{r \in R, r^{2}=r\right\}$. Define the two binary operation + , $\therefore$ on $S$ as follows:

$a+` b=a+b-2 a b$

$$
a \cdot^{`} b=a \cdot b \text { for all } a, b \in S,
$$

then $\left(S,+^{\prime},{ }^{`}\right)$ is a Boolean ring.

Note that any Boolean ring is a commutative regular ring.

Now, we prove the following result. 


\section{Theorem 2.9:}

If $M$ is a maximal ideal in $R$, then $M \cap S$ is a maximal ideal in $S$.

\section{Proof:}

Let $M \cap S$ is not a maximal ideal in S, then there exists an ideal $J$ in $S$ such that $M \cap S \subset J \subseteq S$, so there exists an element $a$ in $J$ and $a \notin M \cap S$, therefore $a \notin M$ and $a \in S$.Since $a=a^{2}$, then $a(1-a)=0 \in M$,

Now ,since $M$ is a prime ideal and $a \notin M$, then $(1-a) \in M$.

$$
\begin{aligned}
(1-a)^{2} & =1-2 a+a^{2} \\
& =1-2 a+a \\
& =1-a
\end{aligned}
$$

So $(1-a) \in S$. Thus $(1-a) \in M \cap S \subseteq J$, also by (2.8)

$1-a+{ }^{\prime} a=1 \in J$ which implies that $J=S$.

Thus $M \cap S$ is a maximal ideal in $S$.

Now, we give the major result of this section .

\section{Theorem 2.10:}

Let $R$ be a regular ring. Then the spectrum topology of $R$ is homeomorphic to the spectrum topology of $S$ which is a subset of all idempotent elements of $R$.

\section{Proof:}

Define a mapping $\Phi$ from $\operatorname{Spec}(R)$ to $\operatorname{Spec}(S)$ by $\Phi(P)=P \cap S$ , for a prime ideal $P$ in $\operatorname{Spec}(R)$. Since $R$ is a regular ring and $P$ is a prime ideal in $R$, so $P$ is a maximal ideal. By Theorem (2.9) $P \cap S$ is a maximal ideal in $S$. Thus $P \cap S$ is a prime ideal in $S$, that is $\Phi(P) \in \operatorname{Spec}(S)$.

To show that $\Phi \square$ is a homeomorphism we must prove that:

1. $\Phi$ is continuous:

Let $U^{*}(e)$ be an open set in $S$. Then

$$
\begin{aligned}
P \in \Phi^{-1}\left(U^{*}(e)\right) & \Leftrightarrow \Phi(P) \in U^{*}(e) \\
& \Leftrightarrow e \notin P \cap S \\
& \Leftrightarrow \Phi^{-1}(e) \notin P, \text { since } e \in S . \\
& \Leftrightarrow P \in U\left(\Phi^{-1}(e)\right),
\end{aligned}
$$


where $U\left(\Phi^{-1}(e)\right)$ is the basic open set of $\operatorname{Spec}(R)$; So $\Phi^{-1}\left(U^{*}(e)\right)=U\left(\Phi^{-1}(e)\right)$ and $\Phi$ is continuous.

2. $\Phi \square$ is injective:

For every prime ideal $P$ of $R$ the image under $\Phi$ of $U(P)$ is $U(\Phi(P))$.

Let $\Phi\left(P_{1}\right)=\Phi\left(P_{2}\right)$, for $P_{1}, P_{2} \in \operatorname{Spec}(R)$, then $P_{1} \cap S=P_{2} \cap S$ which implies that $P_{1}=P_{2}$.

3. $\Phi \square$ is surjective:

The mapping $\Phi$ is surjective since for all prime ideal $P \cap S$ in $\operatorname{Spec}(S)$ there exists a prime ideal $P$ in $\operatorname{Spec}(R)$ such that $\Phi(P)=$ $P \cap S$.

4. $\Phi \square$ is open:

Now, if $a \in R, \Phi(a) \in \Phi(P)$ if and only if $a \in P$ then if $P$ is a prime ideal of $R$ does not contain $a$ then $P \cap S=\Phi(P)$ does not contain $\Phi(a)$ and hence $\Phi(U(a))=U(\Phi(a))$.

This implies that the image of any open subset of $\operatorname{Spec}(R)$ under $\Phi$ is an open subset of $\operatorname{Spec}(S)$. So $f$ is open.

\section{Irreducible Spectrum of Regular Rings:}

This section is devoted to exhibit several properties of irreducible spectrum of regular ring.

We begin this section by the following definition:

\section{Definition 3.1:[4]}

Let $I$ be an ideal of the ring $R$. Then $I$ is said to be irreducible if it is not a finite intersection of ideals of $R$ properly containing $I$; otherwise, $I$ is termed reducible.

\section{Proposition 3.2:[8]}

Let $I$ be a proper ideal of $R$. Then the ideal $\sqrt{I}$ is irreducible if and only if $V(I)$ is irreducible in $\operatorname{Spec}(R)$.

Now, we give the following Proposition which is due to in Lemmermeyer [10]. 


\section{Proposition 3.3:}

Let $R$ be a ring. Then

1) For any pair of ideals $I, J$ in $R$ we have

$V(I \cap J)=V(I J)=V(I) \cup V(J)$

2) $V(I) \subseteq V(J)$ if and only if $\sqrt{I} \supseteq \sqrt{J}$

\section{Corollary 3.4:}

Let $I$ be a proper ideal of regular ring. Then $I$ is irreducible if and only if $V(I)$ is irreducible in $\operatorname{Spec}(R)$.

\section{Proof:}

Let $V(I)$ be irreducible, then by Proposition (3.2) we have $\sqrt{I}$ is irreducible. Since $R$ is regular then by Proposition (2.2) $\sqrt{I}=I$, that is $I$ is irreducible.

Conversely, let $I$ is irreducible. If $V(I)$ is reducible then we can write $V(I)=V\left(I_{1}\right) \cup V\left(I_{2}\right)$.By Proposition (3.3) we have $V\left(I_{1}\right) \cup V\left(I_{2}\right)=V\left(I_{1} \cap I_{2}\right)$, then $V(I)=V\left(I_{1} \cap I_{2}\right)$ that is $I=I_{1} \cap I_{2}$, contradiction, so $V(I)$ is irreducible.

\section{Theorem 3.5:}

If $R$ is a regular ring, then $X=\operatorname{Spec}(R)$ is irreducible if and only if $R$ is an integral domain.

\section{Proof:}

Let $R$ be an integral domain . To show that $\operatorname{Spec}(R)$ is irreducible we must prove that for each pair of elements $a, b \in R$ we have $U(a) \neq \phi$ and $U(b) \neq \phi$ implies that $U(a b)=U(a) \bigcap U(b) \neq \phi$.

Now, if $U(a) \bigcap U(b)=\phi$ then $U(a b)=\phi$ which implies that $a b=0$, therefore either $a=0$ or $b=0$ that is $U(a)=\phi$ or $U(b)=\phi$ contradiction. Hence $U(a) \bigcap U(b) \neq \phi$.

Conversely, let $\operatorname{Spec}(R)$ is irreducible then the nil radical of $R, N(R)$ is a prime ideal therefore $R / N(R)$ is an integral domain. But $R$ is regular then $N(R)=0$, therefore $R$ is an integral domain.

\section{Proposition 3.6:[9]}

The $\operatorname{Spec}(R)$ is irreducible if and only if the nil radical $N(R)$ is a prime ideal. 
Now, we recall the following lemma which is due to in Lemmermeyer [10].

\section{Lemma 3.7:}

The closure of a prime ideal $P$ is $V(P)$. In particular, a prime ideal $P \in \operatorname{Spec}(R)$ is closed (in the Zariski topology) if and only if $P$ is maximal.

Before closing this section we give the following Theorem:

\section{Theorem 3.8:}

The following are equivalent:

1. $\operatorname{Spec}(R)$ is irreducible.

2. $\operatorname{Spec}(R)$ has a generic point.

3. The nil radical of $R$ is a prime ideal.

\section{Proof:}

$1 \rightarrow 3$

By Proposition (3.6) we get $\operatorname{Spec}(R)$ is irreducible if and only if $N(R)$ is a prime ideal.

$3 \rightarrow 2$

Let $N(R)$ be a prime ideal then $N(R) \in \operatorname{Spec}(R)$. Now, let $Q$ be a point in $\operatorname{Spec}(R)$ that is $Q$ is a prime ideal. Since $N(R)=\{\bigcap P: P$ is a prime ideal $\}$, then $N(R) \subset Q$ so $Q \in V(N(R))$, therefore $\operatorname{Spec}(R) \subset$ $V(N(R))$.

But $V(N(R)) \subset \operatorname{Spec}(R)$, therefore $\operatorname{Spec}(R)=V(N(R))$ then by definition of generic point, we have $N(R)$ is a generic point.

$2 \rightarrow 1$

Let $P \in \operatorname{Spec}(R)$ be a generic point then $V(P)=\operatorname{Spec}(R)$.

Assume that $\operatorname{Spec}(R)=A \cup B$ where $A, B$ are closed sets. Let $P \in A \cup B$ then $P \in A$ or $P \in B$.

First let $P \in A$ then $\bar{P}$ the closure of $P$ is the intersection of all closed sets containing $P$. So $\bar{P} \subset A$ since $A$ is a closed set.

By Lemma (3.6), we have $V(P)=\bar{P}$, so $V(P) \subset A$, but $V(P)=\operatorname{Spec}(R)$ therefore $\operatorname{Spec}(R)=A$.

Also in the same way we have $\operatorname{Spec}(R)=B$ that is $\operatorname{Spec}(R)$ is irreducible. 


\section{Connectedness and Compactness:}

This section is concerned with the connectedness and compactness. We prove some results on regular rings and Boolean rings.

\section{Definition 4.1:[2]}

Let $X$ be a topological space. Then $X$ is called totally disconnected if for each pair of distinct points $P$ and $Q$ of $X$, there exists two open subsets $A$ and $B$ of $X$ with the following properties:

$P \in A, Q \in B, A \cup B=X, A \cap B=\phi$

Recall that a topological space is connected if there is no non-empty open sets $\mathrm{A}$ and $\mathrm{B}$ such that

$A \cup B=X$ and $A \bigcap B=\phi$

\section{Theorem 4.2:}

Let $a$ be an idempotent element of $R$. Then $U(a) \cup U(1-a)=\operatorname{Spec}(R)$ and $U(a) \bigcap U(1-a)=\phi$.

\section{Proof:}

Assume that $P$ is a prime ideal in $R$, such that $P \notin U(a)$, therefore $a \in P$ and $1-a \notin P$ since there is no prime ideal contains both $a$ and 1-a. that is $P \in U(1-a)$ and so $U(a) \cup U(1-a)=\operatorname{Spec}(R)$.

Now, let $U(a) \bigcap U(1-a) \neq \phi$, so there exists a prime ideal $P$ in $R$ such that $P \in U(a)$ and $P \in U(1-a)$ therefore $1-a \notin P$ and $a \notin P$, so , a contradiction. $a-a^{2}=0 \notin P$ that is $a(1-a) \notin P$

Hence $U(a) \bigcap U(1-a)=\phi$.

\section{Corollary 4.3:}

If $R$ is a Boolean ring, then for all $a \in R$

$U(a) \cup U(1-a)=\operatorname{Spec}(R)$

$U(a) \bigcap U(1-a)=\phi$

\section{Corollary 4.4:}

If $R$ is a Boolean ring, then $\operatorname{Spec}(R)$ is totally disconnected.

\section{Corollary 4.5:}

If $R$ is a Boolean ring, then $\operatorname{Spec}(R)$ is reducible.

We show that $\operatorname{Spec}(R)$ is disconnected if and only if $R$ contains an idempotent other than 0,1 . To obtain this the following lemma is needed. 


\section{Lemma 4.6:[7]}

Let $R$ be a ring, $N$ is a nil ideal in $R$, and $\bar{a}=a+N$ an idempotent element of $\bar{R}=R / N$. Then there exists an idempotent $e$ in $R$ such that $\bar{e}=\bar{a}$. Moreover, $e$ is unique.

Bourbaki [3] , has shown that for the prime Spectrum of a ring $R$ to be connected, it is necessary and sufficient that $R$ contains no idempotents other than 0 and 1 . Now, we are going to give a different proof of that theorem .

\section{Theorem 4.7:}

Let $R$ be a ring. Then the following conditions are equivalent:

a) $X=\operatorname{Spec}(R)$ is disconnected;

b) $R \cong R_{1} \times R_{2}$ for rings $R_{1}, R_{2}$, neither of which is the zero ring;

c) $R$ contains an idempotent other than 0 and 1 .

\section{Proof:}

$a \Rightarrow b$

Let $V(I), V(J)$ be a non-empty closed sets such that $X=V(I) \bigcup V(J)$ and $V(I) \bigcap V(J)=\phi$ then there is no prime ideal contains both $I$ and $J$. Therefore $R=I+J$ that is $I$ and $J$ are co-prime, and so there exists an element $a \in I$ with 1- $a \in J$. By Proposition (3.3) we have $V(I \cap J)=V(I) \bigcup V(J)$. But $V(I) \bigcup V(J)=X$ so $V(I \cap J)=X$, that is every prime ideal contains and so $I \cap J$ $I \cap J \subseteq N(R)$

But, therefore $I J \subseteq N(R)$ then $I J \subseteq I \cap J$ $a(1-a)=a-a^{2} \in N(R)$. This implies that is an idempotent. $a+N(R)=a^{2}+N(R) \in R / N(R)$

By Proposition (4.6) there exists an idempotent $e$ in $R$ such that is a unit but $a$ is a unit, then $e$. If $e-a \in N(R))$, that is $R(N \bmod e \equiv a$ this is not possible since is not a unit.e -also $1 I \neq R$

Now, we define a homomorphism $f: R \rightarrow R /(e) \times R /(1-e)$ by 
$f(a)=(a+(e), a+(1-e))$ then by Chinese Remainder Theorem we have that $f$ is surjective. $\operatorname{Ker}(f)=(e) \bigcap(1-e)=0$, therefore $f$ is isomorphism, that is $R \cong R_{1} \times R_{2}$.

$b \Rightarrow c$

Since $R \cong R /(e) \times R /(1-e)$, then the element

$(1,0) \in R /(e) \times R /(1-e)$ is a non-zero idempotent.

$c \Rightarrow a$

Let $e \in R$ be an idempotent other than 0 and 1 .

Let $X=V(e) \cup V(1-e)$ and $V(e) \cap V(1-e)=\phi$.

Since $e(1-e)=0 \in P$, then every prime ideal contains either $e$ or 1-e. If $V(e)=\phi$ then $e$ is a unit, but then $e=1$, also if $V(1-e)=\phi$ then $1-e=1$ and $e=0$. Contradiction, therefore $V(e), V(1-e)$ are non-empty closed sets.

Proposition 4.8:[5]

If $\mathrm{R}$ is regular, then every prime ideal is maximal.

Before closing this section, we present the following result.

\section{Theorem 4.9:}

\section{Proof:}

If $\mathrm{R}$ is regular ,then every point in $\operatorname{Spec}(\mathrm{R})$ is closed.

Since $\mathrm{R}$ is a regular ring, then by Proposition (4.8) we have every prime ideal is maximal . That is every point in $\operatorname{Spec}(\mathrm{R})$ is maximal.

Recall Lemma (3.7) we get that every point in $\operatorname{Spec}(R)$ is closed. 


\section{REFERENCES}

[1] Alkam, O.K. (1987) “On Regular Ring”, M. Sc. Thesis, University of Jordan .

[2] Behrens, E.A. (1972) "Ring Theory", McMaster University, Canada, Hamilton, Outario .

[3] Bourbaki, N. (1972) Elements of Mathematics, Commutative Algebra, Great Britain, Addison-Wesley Publishing Company.

[4] Burton, D.M. (1970) A First Course in Rings and Ideals, Addison Wesley Publishing Company.

[5] Fisher, J. W. (1974) Von Neumann Regular Rings Versus V-Rings, Ring Theory", Proc. Oklahoma Conference, Lecture Notes in Pure and App L. Math. 7, Dekker, PP.101-119.

[6] Heinzer, W. and Roitman M. (1997) "The Homogeneous Spectrum of a Graded Commutative Ring”, American Mathematical Society, http://www.math.purdue.edu/ heinzer/preprints/homog42.pdf

[7] Jacobson, N. (1980) Basic Algebra II, United States of America ,Allyn and Bacon, Inc.

[8] Jao, D. (1999) Commutative Algebra, www.dominiaOrg/djao/ Algebra, pdf.

[9] Laksov, (2002) "Algbook The Zariski Topology”, www.math.kth.Sc / Laksov/courses/algebra adr 01/notes/Rings 5.

[10] Lemmermeyer, F. (2003) "The Spectrum of a ring" ,www.fen bjLkent.Edu.tr/ Franz/Alge.

[11] Ming, R.Y.C. (1974) "On Von Neumann Regular Rings", Proc. Edinburgh Math. Soc. 19, PP. 89-91.

[12] Ming, R.Y.C. (1980) "On Von Neumann Regular Rings”, V, Math. J. Okayama Univ. 22, PP.151-160.

[13] Ming, R.Y.C. (1983) "Maximal ideals in Regular rings", Hokkaido Mathematical Journal, Vol. 12, PP.119-128.

[14] Warrenb, (2001) "Commutative Rings and Integral Domains", www.math.bsuedu/ warrenb/courses/Ringspdf. 